\title{
Detailed investigation of aerothermal behaviour of confined impinging jet
}

\author{
by J.-M. Buchlin and M. Laperches
}

von Karman Institute for Fluid Dynamics, Rhodes-Saint-Genèse, Belgium

\begin{abstract}
The detailed distribution of the convective heat transfer coefficient in the vicinity of the impingement of a vertical turbulent circular air jet on a horizontal flat plate is inferred from temperature measurements performed by quantitative infrared thermography. Steady-state experiments are conducted with the heated-thin-foil method. PIV measurements performed by seeding the air jet with smoke and illuminating the section of interest by a YAG laser sheet support the interpretation of the IR results. The effect of the jet Reynolds number ranging from 20000 to 70000 , the normalised stand-off distance with values as small as 0.1 and the presence of a roofing on the thermal exchange coefficient is emphasized. It is shown that two heat-transfer peaks can develop in the impingement region. Their magnitude and position change with the stand-off distance and are well correlated to the flow features.
\end{abstract}

\section{Nomenclature}

$\begin{array}{lll}D & \text { Exit nozzle diameter } & \varepsilon \\ h & \text { Heat transfer coefficient } & v \\ k & \text { Thermal conductivity } & \\ N u & \text { Nusselt number } & \\ r & \text { Radial distance } & \\ R e & \text { Reynolds number } \\ T & \text { Temperature } \\ U & \text { Velocity } \\ Z & \text { Stand-off distance } & \end{array}$

Greek symbols

Emissivity factor

Kinematic viscosity

$\checkmark$

(1)

(1)

\section{Introduction}

The wide application of impinging fluid jets in industrial processes is justified by the high heat and/or mass transfer rates achieved within such systems. Typical examples are the annealing of metals, the tempering of glass sheets, the drying of paper and textiles, the cooling of turbine blades and the anti-icing of aircraft. In some situations, the jet expands in a confined space either because the stand-off distance of the nozzle exit with respect to the solid surface is small or because a roofing exists around the jet. Such circumstances may affect the jet development and modify the impingement conditions so that the heat transfer differs from the case of an unconfined jet.

The present paper deals with this question in describing an experimental investigation relying on the application of the quantitative infrared thermography (QIRT) and particulate image velocimetry (PIV) techniques. The suitability of such non-intrusive techniques to retrieve the local features of impinging jets is demonstrated. This study is carried out in the frame of the VKI research programme addressed to the detailed description of the aerothermal behaviour of such complex flow [1-3] with the aim to built-up reliable data bases to support and validate dedicated CFD methods such as LES or DNS.

After presenting the main features of the experimental set-up and the procedure followed to collect the PIV and QIRT data, the paper emphasises the effect of the jet Reynolds number $R e$, the normalised stand-off distance of the nozzle $Z I D$ and the presence of a roofing around the jet on the distribution of the convective heat transfer coefficient. A correlation is sought between the flow field and the thermal distribution at the wall. 


\section{Experimental setup}

The experimental test set-up is sketched in figure 1a. A close view of the test section is proposed in figure $1 \mathrm{~b}$. It consists of an horizontal $0.5 \mathrm{~m}$ diameter flat plate made of a thin copper foil, $40 \mu \mathrm{m}$ thick, chemically treated to produce a double spiral circuit $1 \mathrm{~mm}$ wide with groves of $2 \mathrm{~mm}$, fixed on an epoxy sheet, $1 \mathrm{~mm}$ thick. The foil is heated by the Joule effect.

A FMV Lamel centrifugal fan produces the airflow. It is located in a caisson with an opening to the atmosphere where smoke is injected to seed the jet. A converging settling chamber of $0.4 \mathrm{~m}$ in diameter ends with a large nozzle of $0.05 \mathrm{~m}$ in diameter. The size of the nozzle is intentionally chosen large to improve significantly the resolution of the impinging zone to be analysed. The jet is at ambient temperature and flows vertically downward. The exit of the nozzle may be coincident with a roofing formed by a circular flate plate of $0.5 \mathrm{~m}$ in diameter also at ambient temperature so that no heat exchange between the jet and the roofing takes place.

Only the rear face of the foil is coated with a black paint of emissivity factor $\varepsilon_{w}=0.95$ to improve the thermography measurements and allow correction for radiation. During measurement, the rear surface viewed by the IR scanner, is surrounded by a black plastic curtain that minimises the heat loss by natural convection at the edges. The IR scanner is the AGEMA Thermovision 900 system with a HgCdTe detector sensitive in the 8-12 $\mu \mathrm{m}$ wavelength range and cooled by liquid nitrogen. The measurable temperature range is $-30^{\circ} \mathrm{C}$ to $1500^{\circ} \mathrm{C}$ with an announced thermal sensitivity of $0.1^{\circ} \mathrm{C}$. The camera is equipped with a standard optical set-up of $10^{\circ}$ vertical $\times 5^{\circ}$ horizontal giving an instantaneous field of view (IFOV) of $0.76 \mathrm{mrad}$. The camera scans the rear of the heated thin foil via a special 45-degrees mirror. A spatial resolution of about 1pixel per millimetre is achieved. It is connected to a dedicated $\mathrm{PC}$ for acquisition at a frame rate of $20 \mathrm{~Hz}$ and processing of thermogrammes (272×136 pixels).

Flow visualisation is obtained by illuminating a vertical plane of the jet with a doublepulsed YAG laser sheet. A TSI cross correlation CCD camera (640x480 pixels) protected from the smoke by a Plexiglas box, scans the field of interest. The YAG laser and the CCD camera are connected via a TSI synchroniser to a computer with INSIGHT software for acquisition and processing of the PIV images. The velocity vectors are deduced from such visualisations obtained for an interpulse period ranging from $50 \mu \mathrm{s}$ to $200 \mu$ s to obtain the optimum particle displacement depending on the Reynolds number.

\section{IR Data restoration}

Calibration of the camera is performed by plotting the camera output in terms of the thermal units, TU, versus the plate temperature measured by a thermocouple flush mounted on the top surface (jet face) of the target. In the working temperature range $20-80^{\circ} \mathrm{C}$, the calibration curve $T-T U$ can be approximated by a quadratic polynomial.

The scanning of the heated flat plate without jet, the case of a pure natural convection where the heat transfer coefficient is constant over the surface, shows a very good heating uniformity over $99 \%$ of the impingement area. The convective heat transfer coefficient is inferred from the temperature of the wall $T_{w}(r)$ by application of the Newton law:

$$
h(r)=\frac{q_{c v}(r)}{T_{w}(r)-T_{r e f}}
$$

where $T_{\text {ref }}$ is a reference temperature taken equal to the jet temperature $T_{j}$ in the present investigation. The convective heat flux $q_{c v}$ is calculated by subtracting the contribution of the radiation $q_{\mathrm{rad}}$ and lateral conduction $q_{\mathrm{cd}}$ to the Joule heating $q_{J}[1-3]$. In such a thin-foil design, the thermal losses by conduction (copper and epoxy) are negligible ( $\leq 1 \%$ ), while the heat loss by radiation does not exceed $5 \%$ of the total flux. 


\section{Typical results}

Experiments are conducted by varying the jet Reynolds number, $R e=U_{j} D / v$, from 20000 to 70000 and the normalised stand-off distance ZID from 0.08 to 5.6. The range of Reynolds number guarantees the turbulent character of the jet. Due to the large nozzle diameter the flow remains highly incompressible; the Mach number does not exceed 0.06. The low ZIDvalues tested confer an additional original character to the study. Since preliminary tests have confirmed the very good axisymmetry of the flow and the subsequent thermal field, only half of the jet is analysed.

\subsection{PIV results}

Figure 2a shows a typical flow visualisation of the jet where turbulent structures are observed. The PIV quantification of such pictures yields the velocity field. A typical instantaneous vector plot, presented in figure $2 b$, shows the flow field for a high Re-value of 60000 and a small ZID-value of 1 in the case of no roofing. The main observations pointed out by the arrows are in very good agreement with the sparse literature on the subject [4-5]. A radial acceleration zone is detected just after the stagnation point in a region located between 0.6 and $1 r / D$. Then a wall jet develops; it is mainly characterised by primary and secondary vortex rings (the latter not very well captured due to light reflection) leading to local flow detachment and ending by a reattachment point found at about $r / D=2.4$ with a good repeatability. The PIV results plotted in figure $2 c$ exemplify the experiments with roofing. The same flow pattern is retrieved indicating that the presence of the roofing does not affect the jet behaviour. In conclusion, the correlation that should exist between the flow and the heat transfer allows to anticipate non uniform temperature distribution in the impingement region.

\subsection{QIRT results}

A typical false-colour IR thermogramme is shown in figure 3 . As expected, it emphasises the non-uniform temperature field with concentric cold and hot rings revealing the presence of high and low heat transfer regions. The local heat transfer is determined from the measurement of a wall temperature profile illustrated in figure 3 and plotted in terms of the local Nusselt number $N u=h D / k_{a}$.

The effect of $R e$ and $Z I D$ on the Nu-distribution is clearly shown in figure 4. At very small

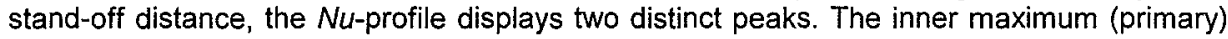
occurs around $r / D=0.7$ while the outer (secondary) is situated close to $r / D=2.4$. These two heat transfer peaks correlate very well with the acceleration and the reattachment zones of the flow. At small stand-off distances, the secondary peak dominates as $R e$ increases. As the stand-off distance increases further and/or the Reynolds number decreases the inner and outer peaks vanish and the maximum heat transfer moves to the stagnation point; there, the distribution takes a conventional bell shape. All these trends are confirmed in figure 5 which, in turn, denotes the particular situation of $Z D<0.1$. In such a case, the outer peak displaces to the centre and tends to merge with the inner peak forming a region of high heat transfer between 0.7 and $1.3 r / D$. Since no PIV data are available for this configuration, it is guessed that the very small gap between the edges of the nozzle and the plate produces a significant flow acceleration, which hinders the vortex ring interaction and restores faster the flow reattachment.

In agreement with the PIV results, the QIRT data shown in figure 5 confirm the weak influence of the roofing since up to $r / D=3$ no significant difference appears between the two series of tests.

To summarise the QIRT data, the position of the peaks is plotted in function of the standoff distance in figure 6a and the magnitude of the heat transfer coefficients at these peaks in figure $6 b$. The primary peak remains well located at $r / D=0.7$ where flow acceleration is detected while the position of the secondary peak which is conjectured to be linked to the flow reattachment evolves; it moves away from the jet axis as $Z / D$ increases to fix its location 
at about $r / D=2.4$ as $Z / D$ becomes larger than 2 . Such a finding agrees with the Lytle and Web data [6]. Below $Z D=2$ the outer peak is the stronger, especially when $R e$ is large; above $Z / D=3$, the heat transfer profile tends to the conventional bell shape.

\section{Conclusions}

QIRT and PIV are two complementary non-intrusive techniques that provide an explanation for the non-uniformity of the heat transfer coefficient in an impinging jet at small stand-off distance. Due to flow acceleration, complex vortex interaction and reattachment, the heat transfer distribution may exhibit two distinct peaks which may be higher than the one at the stagnation point. The effect of $R e$ and $Z / D$ on the location of these peaks is identified. The present data are in agreement with the literature.

\section{REFERENCES}

[1] BUCHLIN (J.-M.) and DUBOIS (M.) - Heat transfer of impinging multijet system. An application of the quantitative thermography. In: Quantitative Infrared Thermography QIRT'92 (Balageas D., Busse G., Carlomagno G. M., eds), 117-120, 1992, Edizioni ETS.

[2] BUCHLIN (J.-M.), PRETREL (H.), PLANQUART (P.), LANGER (H.), THIRY (F.) - Infrared thermography study of a thermal anti-icing system. In: Quantitative Infrared Thermography QIRT 94 (Balageas D., Busse G., Carlomagno G. M., eds), 133-138, 1994, Edizioni ETS.

[3] BUCHLIN (J.-M.), MEYERS (M.) - Infrared thermography study of a confined impinging circular jet. In: Quantitative Infrared Thermography QIRT 96 (Balageas D., Busse G., Carlomagno G.M., eds), 129-134, 1996, Edizioni ETS.

[4] LANDRETH (C.C.), ADRIAN (R. J.) - Inpingement of a low Reynolds number turbulent circular jet onto a flat plate at normal incidence. In: Experiments in Fluids 9, 74-84, 1990.

[5] MEOLA (C.), LUCA (L.), CARLOMAGNO (G. M.) - Influence of shear layer dynamics on impinging heat transfer. In: Experimental Thermal and Fluid Science 13, 29-37, 1996.

[6] LYTLE (), WEBB () - Secondary heat transfer maxima for air jet impingement at low nozzle-to-plate spacings. In: Experimental heat transfer, Fluid Mechanics, and Thermodynamics, 1991.

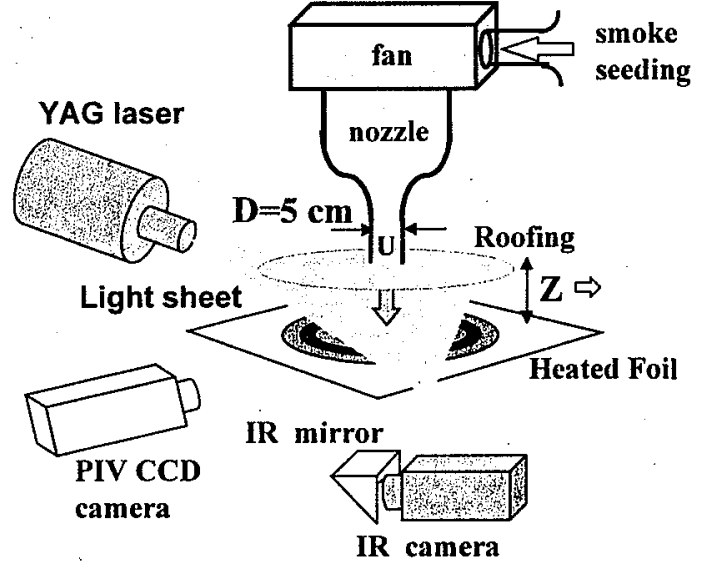

(a)

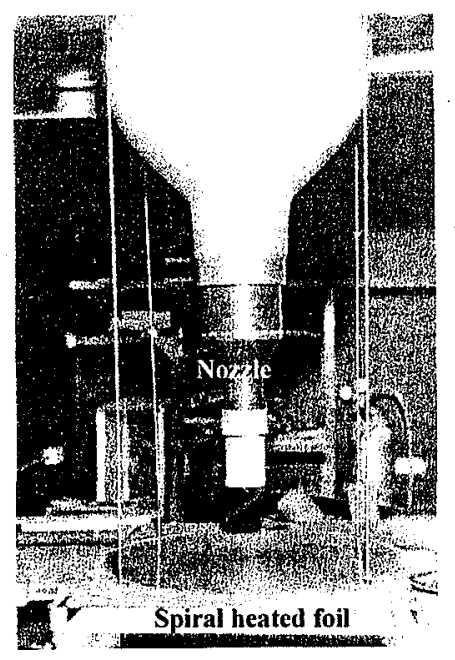

(b)

Fig. 1. Schematic of the test setup (a) and close view of the test section (b) 
http://dx.doi.org/10.21611/qirt.1998.041

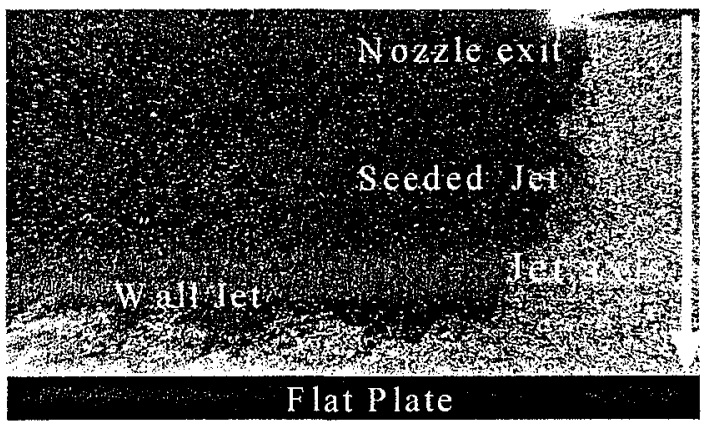

(a)

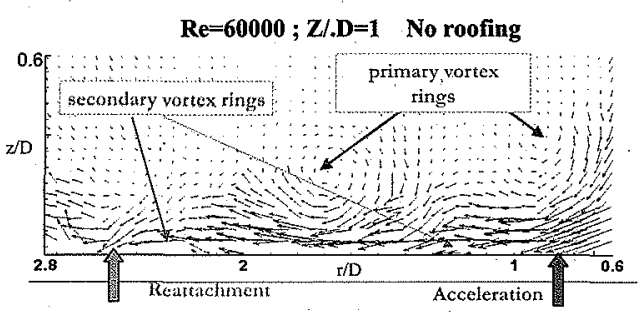

(b)

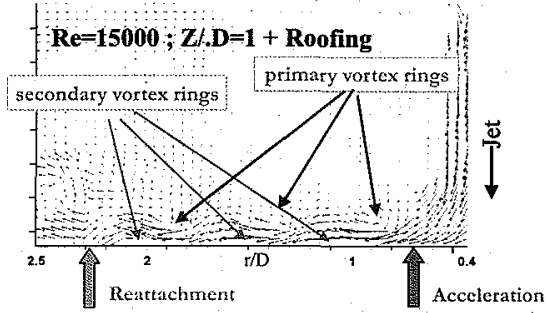

(c)

Fig. 2. Flow visualisation and PIV results

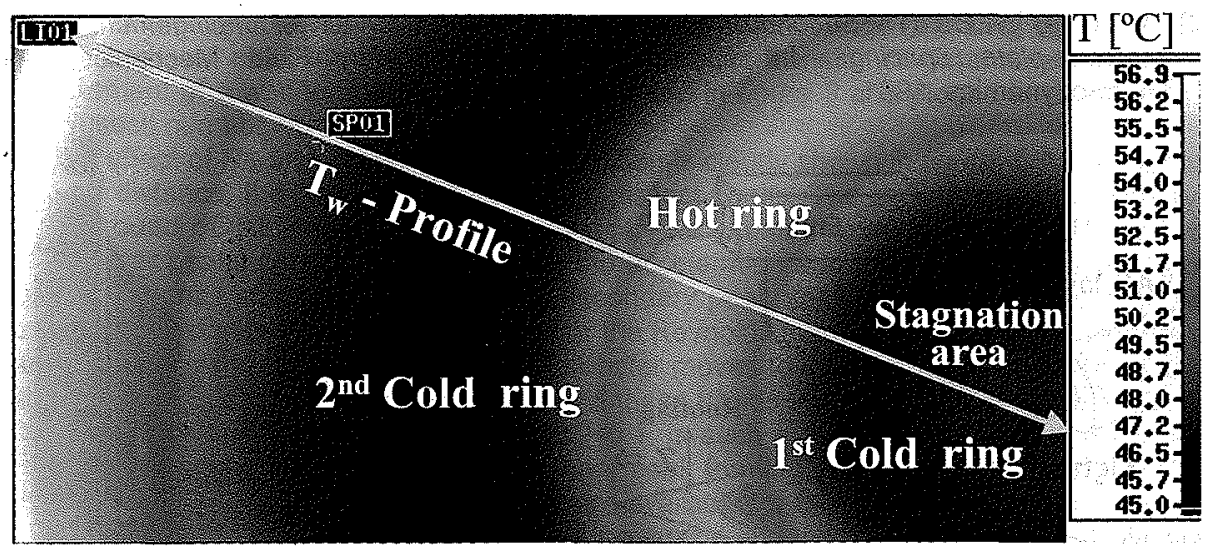

Fig. 3. Typical IR thermogramme of the impingement region 
http://dx.doi.org/10.21611/qirt.1998.041
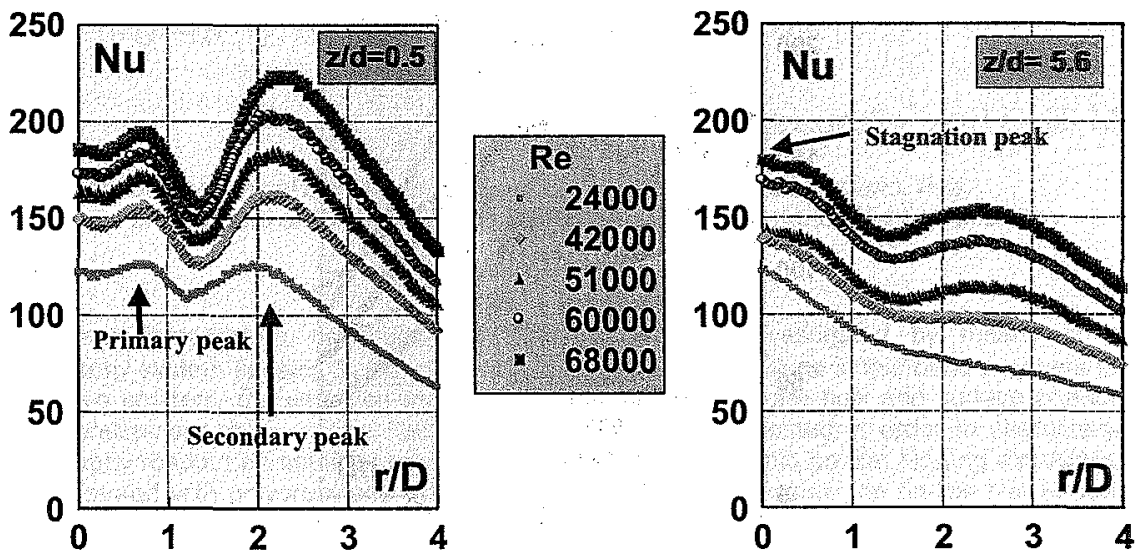

Fig. 3. Effect of $R e$ on the $\mathrm{Nu}$-distribution: no roofing
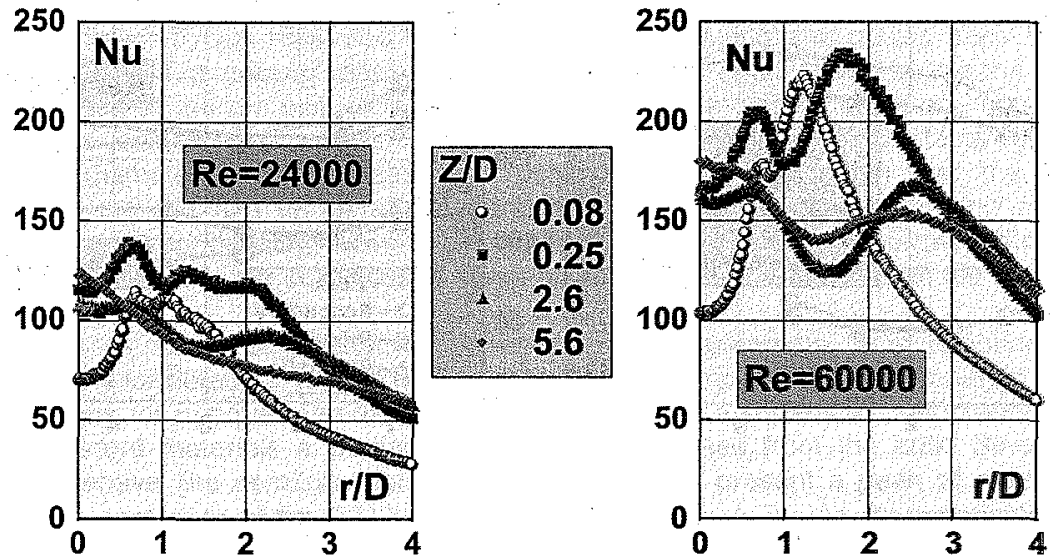

Fig. 4. Effect of $Z / D$ on the Nu-distribution: no roofing 
http://dx.doi.org/10.21611/qirt.1998.041

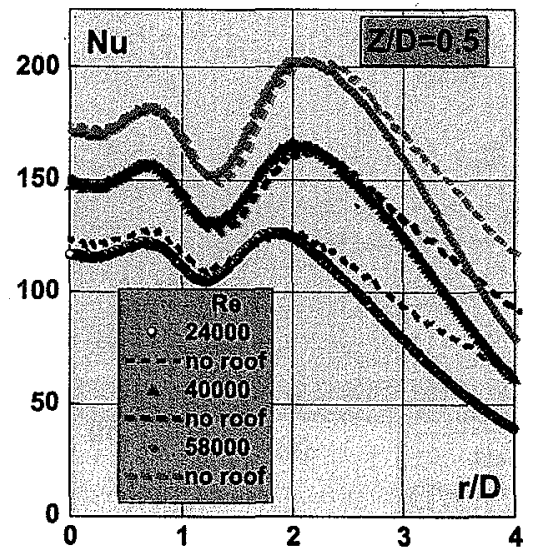

Fig. 5. Effect of the roofing on the Nu-distribution: no roofing

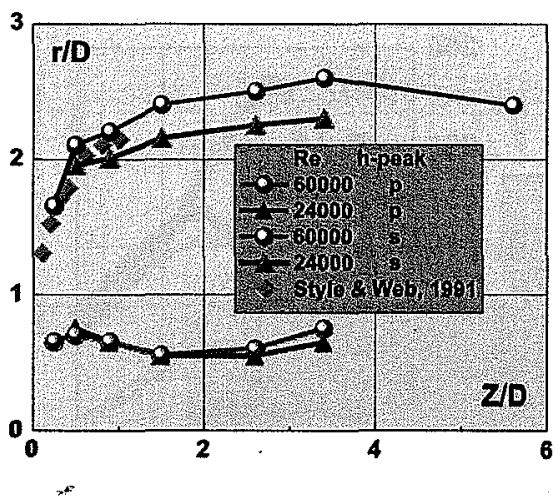

(a)

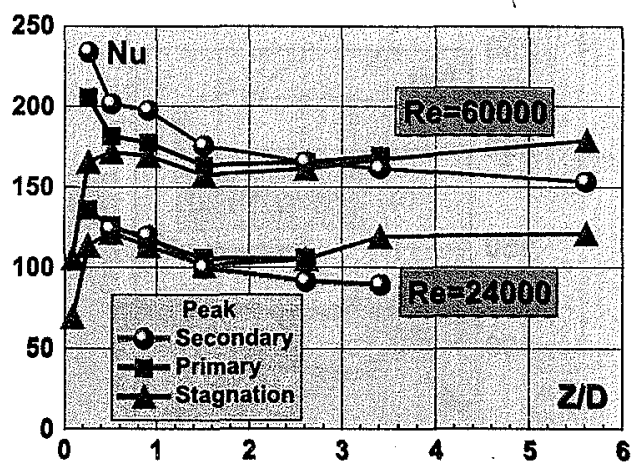

(b)

Fig. 6. Effect of $Z / D$ on the Nu-distribution: no roofing 